\title{
Tekmeria
}

Vol 10 (2011)

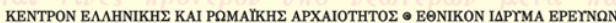
CENTRE DE RECHERCHE DE L'ANTIOUTTE GRECQUE ET ROMAINE FONDATON NATOOMAE DE LA RECH RCHE SCIINTIFOOU

\section{Hellanodiken außerhalb von Olympia}

S. B. ZOUMBAKI

doi: $\underline{10.12681 / \text { tekmeria.266 }}$

\section{$T \epsilon \kappa \mu \eta \dot{\rho} \iota \alpha$}

СYMBOAES ETHN IETOPA TOY EAMHIKOO KAL PQMAIKO KOEMOY • CONTRIBUTIONS TO THE HISTORY OF THE GREEK AND ROMAN WORLD • CONTRIBUTION A L'HISTOIRE DU MOVDE GREC ET ROYMIN - BETRAGE ZUR GESCHICHTE DER GRIECHISCHEN UND ROMISCHEN WEUT

\section{0}

A๑HNA 2011

DIFFUSION DE BOCCARD - 11, RUE DE MEDICIS, 75006 PARIS

\section{To cite this article:}

ZOUMBAKI, S. B. (2011). Hellanodiken außerhalb von Olympia. Tekmeria, 10, 7-21.

https://doi.org/10.12681/tekmeria.266 


\section{Hellanodiken außerhalb von Olympia*}

Die Bezeugungen von Hellanodiken in Zusammenhang mit Agonen außerhalb von Olympia haben L. Robert und P. Charneux kurz besprochen und einige dieser Belege erwähnt. ${ }^{1}$ Es überrascht nicht, dass das Hellanodikenkollegium in der Kaiserzeit im Rahmen von athletischen Veranstaltungen vorkommt, die den Status der Olympischen oder Isolympischen Spiele erhielten und somit auch bisweilen den Kampfrichtertitel imitierten. Die Präsenz von Hellanodiken außerhalb von Olympia in früheren Epochen ist jedoch anscheinend auf einer anderen Basis zu untersuchen und interpretieren, was die Aufgabe dieser Arbeit darstellt.

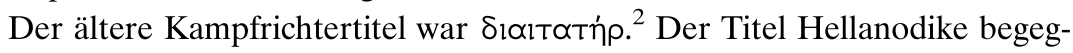

\footnotetext{
* Prof. P. Siewert und Prof. M. Hatzopoulos verdanke ich nützliche Diskussionen und wertvolle Hinweise und $\mathrm{Cl}$. Lepeniotis die sprachliche Verbesserung meines Textes.

Folgende Abkürzungen werden verwendet:

Buraselis, Das hellenistische Makedonien: K. Buraselis, Das hellenistische Makedonien und die Ägäis (München 1982).

Gabbert, Antigonus II Gonatas: J. Gabbert, Antigonus II Gonatas. A political biography (London-New York 1997).

$L S A G^{2}$ : L. Jeffery, The local scripts of Archaic Greece, Revised edition with a Supplement by A.W. Johnston (Oxford 1990).

Perlman: P. Perlman, City and sanctuary in ancient Greece (Göttingen 2000).

1. L. Robert, Hellenica V (1948) 59-63; Hellenica VIII (1950) 80; P. Charneux, «Inscriptions d'Argos», BCH 80 (1956) 608-610.

2. Zum Titel Diaitater s. J. Ebert $\mathcal{E}$ P. Siewert, «Eine archaische Bronzeurkunde aus Olympia mit Vorschriften für Ringkämpfer und Kampfrichter», OlB 11 (1999) 391-412 (SEG XLVIII 541) und zwei noch unedierten Inschriften auf Bronzetafeln Inv. Nr. B 1291 und B 6901, die auch entsprechend in P. Siewert, «The Olympic rules», in: W. Coulson $\mathfrak{E} \mathrm{H}$. Kyrieleis (Hrsgg.), Proceedings of an international symposium on the Olympic games, 5-9 September 1988 (Athen 1992) 115 und ders., «Symmachien in neuen Inschriften von Olympia», in: L. Aigner Foresti et al. (Hrsgg.), Federazioni e federalismo nell'Europa antica. Bergamo, 21 25 settembre 1992 (Milano 1994) 257 Anm. 2 erwähnt werden; vgl. auch P. Siewert, «'Richter
} 
net zum ersten Mal in der 3. Olympischen Ode Pindars auf den Olympiasieg Therons von Akragas $476 \mathrm{v}$. Chr. Um diese Zeit ist auch die erste epigraphische Bezeugung des Terminus in einer Inschrift von Olympia des Zeitraums 475-450 v. Chr. zu finden. ${ }^{3}$ Was die genaue Zeit und den Anlass dieser Umbenennung betrifft, argumentiert P. Siewert aufgrund einer 1965 in Olympia aufgefundenen Inschrift, daß die elischen Schiedsrichter (Diaitateres) in der Zeit zwischen der Schlacht von Plataiai (479 v. Chr.) und den Olympien von 476 v. Chr. zu "Hellenenrichter" (Hellanodikai) umbenannt wurden. ${ }^{4}$ Der Wechsel von der Bezeichnung Diaitater

über die Hellenen' (Hellanodikas) und andere überstaatliche Gemeinschaftsbezeichnungen in Olympia», in: M.-G. Angeli Bertinelli \& A. Donati (Hrsgg.), Il cittadino, lo straniero, il barbaro, fra integrazione ed emarginazione nell' antichità, Atti del I Incontro Internazionale di Storia Antica, Genova 22-24 maggio 2003, Serta Antiqua et Medievalia VII (Roma 2005) 93-94, Anm. 4.

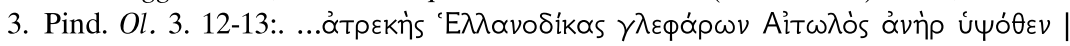

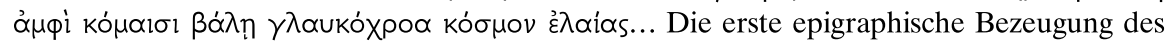
Hellanodikentitels ist in $I v O 2$ 2, die nach $L S A G^{2}, 218-220$, Nr. 15 zwischen 475-450 v. Chr. datiert wird. Diese Datierung ist allgemein angenommen, während der alte Chronologievorschlag von A. Kirchhoff, $A Z 38$ (1880) 66, Nr. 362 um 580 v. Chr. nicht mehr haltbar ist.

4. P. Siewert, «L'autonomie de Hyettos et la sympolitie thespienne dans les Helléniques d'Oxyrhynchos», REG 90 (1977) 463 (SEG XXVI 475); P. Roesch, «Epigraphica 1978», Teiresias, 1978, 14, no. E 78. 20 (SE G XXVIII 426); P. Siewert, «Eine Bronze-Urkunde mit elischen Urteilen über Böoter, Thessaler, Athen und Thespiai», OlB 10 (1981) 228-248 Taf. 24 (SEG XXXI 358); $L S A G^{2}$, 450, Nr. E; H. van Effenterre \& Fr. Ruzé, Nomima. Recueil d'inscriptions politiques et juridiques de l'archaïsme grec, I (Rome 1994) 248-249, Nr. 60; P. Siewert, «Richter über die Hellenen» (a.O. Anm. 2) 93-104.

Die Inschrift enthält die Revision eines früheren Urteils von Menandros und Aristolochos seitens der elischen Aufsichtsbehörde der Mastroi zugunsten von Athen und Thespiai und zu Lasten der Böoter und Thessaler. Es geht wahrscheinlich um die Ereignisse nach der Schlacht von Thermopylai, da Xerxes mit der Hilfe von thessalischen und böotischen Kontigenten im Sommer $480 \mathrm{v}$. Chr. Thespiai und Athen verwüstete. So werden die Perserfreunde für den Bruch der olympischen Ekecheirie aufgrund des olympiakos nomos nach der Schlacht von Plataiai verurteilt. Da die zwei Richter, Menandros und Aristolochos, nur namentlich erwähnt werden und ihre Entscheidung von den Mastroi revidiert wurde, nimmt P. Siewert an, dass es sich um Hellanodiken handelt - bekanntlich von den Mastroi kontrolliert-, die in diesem Fall tatsächlich über die propersischen Hellenen (Böoter und Thessaler) und die antipersischen Hellenen (Athener und Thespier) richteten. Dass die zwei Personen als Hellanodiken zu betrachten sind, bezweifelt M. Lämmer, «La cosidetta "pace olimpica" nell'antiquità greca», in: P. Angeli Bernandini (Hrsg.), Lo sport in Grecia (Bari 1988) 149, Anm. 66. Zu den Behörden der griechischen Städte, die die Amtsinhaber kontrollierten, s. P. Fröhlich, Les cités grecques et le contrôle des magistrats (IVe-Ier siècle avant J.C.) (Genève 2004) und besonders für Elis 190-191. 
zum anspruchvolleren Titel Hellanodikes entspricht der Erhebung der elischen Kampfrichter zu panhellenischen Richtern, was sicher nicht ohne Zustimmung oder sogar Anstiftung der Schutzmacht Sparta geschah. ${ }^{5}$

Im Lauf der Zeit blieben die Hellanodiken von Olympia, die Wächter der Ekecheirie. Sie waren hochangesehene Funktionäre nicht nur als Kampfrichter im agonistischen Bereich, die auch über die hellenische Abstammung der Athleten entschieden, sondern auch im rechtlichen Bereich allgemein. Die Hellanodiken waren zuständig für mehrere Fälle, die direkt oder indirekt mit der Rechtsprechung zu tun hatten. ${ }^{6}$ Auf das enge Verhältnis zwischen Recht und Agonen wurde schon von Historikern und Rechtshistorikern hingewiesen. ${ }^{7}$ Zahlreiche Beispiele bezeugen, dass die Entscheidungen der Hellanodiken panhellenischen Respekt genossen. ${ }^{8}$ Sie galten als "panhellenisches Gericht", wie es mehrere Jahrhunderte später

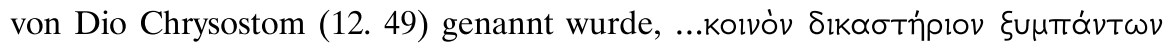

5. Die Gültigkeit des olympiakos nomos wurde von den Spartanern nicht in Abrede gestellt, auch nicht zur Zeit der Entfernung der Eleer von ihrer Symmachie, als eine Strafe von 2000 Minen bei den Spartanern selbst vom olympiakos nomos durchgesetzt wurde, s. Thuk. 5. 49, 1-50, 4; vgl. J. Roy, «Thucydides 5. 49, 1-50, 4: the quarrel between Elis and Sparta in 420 B.C., and Elis' exploitation of Olympia», Klio 80, 1998. 2, 360-68.

6. Vgl. z.B. die sog. "stèle de sûla" von Kyrene, die eine Liste von Auslösungen von verschiedenen gegen Kyrene proklamierten syla enthält, wobei die jeweiligen Behörden der betreffenden Städte genannt werden, die die diesbezüglichen Dokumente aufbewahrten; als Vertreter des elischen Staates waren die Hellanodiken von Olympia dafür zuständig: G. Pugliese Caratelli, «Supplemento epigrafico Cirenaico», ASAA 23-24 (1961-62) 273-280, Nr. 103, Abb. 81; G. Daux, «Note sur une inscription de Cyrène», BCH 87 (1963) 388390; B. Bravo, «Sulân. Représsailles et justice privée contre les étrangers dans les cités grecques», ASNP 10.3 (1980) 918-926; ferner vgl. Y. Garlan, «Études d'histoire militaire et diplomatique, II: Sur le règlement des droits de représsailles», BCH 89 (1965) 338-339; $\mathrm{Ph}$. Gauthier, Symbola. Les étrangers et la justice dans les cités grecques (Nancy 1972) $213 \mathrm{ff}$.

7. Vgl. z.B. die Untersuchung über den Terminus brabeus, J. Velissaropoulou, «Justice and games: the brabeus», in: R. W. Wallace \& M. Gagarin (Hrsgg.), Symposion 2001. Vorträge zur griechischen und hellenistischen Rechtsgeschichte, Evanston, Illinois, 5.-8. September 2001 (Wien 2005) 305-315 mit diesbezüglicher Literatur.

8. Vgl. z.B. die Erzählungen von Pausanias (5. 21, 5-6) und Plutarch (Vitae decem oratorum 850 B) bezüglich des Athleten Kallippos aus Athen; vgl. I. Weiler, «Korruption in der olympischen Agonistik und die diplomatische Mission des Hypereides in Elis», in: A.D. Rizakis (Hrsg.), Achaia und Elis in der Antike, Akten des 1. Internationalen Symposiums, Athen,

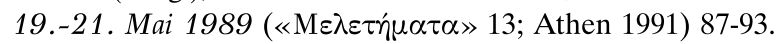

Vgl. ferner die Inschrift des Denkmals des Troilos, der zugleich Olympionike und Hellanodike war (IvO 166); dass er Hellanodike war, wird als 'E $\lambda \lambda \eta \dot{\eta} \nu \omega \nu \tilde{\tilde{p}} \rho X 0 \nu$ Tót' 'O $\lambda \cup \mu-$ ríạ ausgedrückt. 


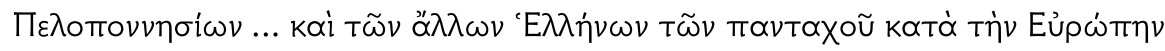

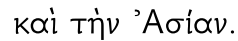

\section{Hellanodiken als Richter bei Xenophon}

Die erste Bezeugung von Hellanodiken außerhalb von Olympia und zwar nicht im agonistischen, sondern im rechtlichen Bereich findet sich bei Xenophon (Lak. Pol. 13. 11). Xenophon zufolge wollte sich der spartanische König auf dem Schlachtfeld ausschließlich mit dem Krieg beschäftigen, so dass er die Schiedssprüche an die Hellanodiken verwies. Der Bericht Xenophons, der keineswegs in Abrede gestellt werden kann, da der Autor selbst am Feldzug von Agesilaos teilnahm, liefert jedoch weder für das Verständnis der Identität der erwähnten Hellanodiken noch für die Zeit der Einführung dieser Praxis in Sparta oder in die Peloponnesische Symmachie irgendwelche Anhaltspunkte. Die von Xenophon erwähnten Hellanodiken wurden in der bisherigen Forschung als spartanische Magistraten interpretiert, die als Schiedsrichter zwischen Griechen innerhalb der Peloponnesischen Symmachie fungierten. ${ }^{9}$ Was die Zeit der vermutlichen Einführung der Institution in die

9. J. Oehler, RE VIII 1 (1912) 155 s.v. Hellanodikai sieht in den von Xenophon erwähnten Hellanodiken "die spartanischen Richter im Heere der peloponnesischen Symmachie“. H. Schaefer, Probleme der alten Geschichte. Gesammelte Abhandlungen und Vorträge (Göttingen 1963) 289 Anm. 4 glaubt, dass die Hellanodiken als Schiedsrichter zwischen Spartanern und den übrigen Griechen auf dem Schlachtfeld im 6. Jh. v. Chr. in die Peloponnesische Symmachie eingeführt wurden, vgl. auch U. Kahrstedt, Griechisches Staatsrecht. I: Sparta und seine Symmachie (Göttingen 1922) 184, Anm. 4; 250; 326 Anm. 2. D.M. Mac Dowell, Spartan Law (Edinburgh 1986) 125 f. und 132 f., nimmt an, daß die Hellanodiken eine schiedsrichterliche Rolle zwischen Spartanern und anderen Griechen sowohl innerhalb Spartas als auch in Feldzügen spielten; da aber Ausländer in Sparta nicht willkommen waren, seien also die Hellanodiken eher auf den Feldzügen aktiv gewesen. Mac Dowell interpretiert ferner die spartanischen Gesandten zum Gericht von Plataiai (427 v. Chr.) und von Ismenias in Theben (388 v. Chr.) trotz des Schweigens der diesbezüglichen Quellen als Hellanodiken (Xen. Hell. 5. 2, 35). L. Thomsen, Lakedaimonion politeia (Stuttgart 1996) 67 nimmt an, dass sowohl die Hellanodiken als auch die ebenso in der gleichen Xenophonstelle erwähnten Tamiai spartanische Magistraten im Rahmen der Peloponnesischen Symmachie waren, deren Aktivität nur in der Situation nach den Perserkriegen einen Sinn hätte; Thomsen bemerkt ferner, dass um die gleiche Zeit auch die Hellanodiken von Olympia erstmals bezeugt sind und dass die Hellanodiken von Sparta keine Kompetenzen in ihrer Heimat innezuhaben scheinen. Nach M. Lipka, Xenophon's Spartan Constitution (Berlin - New York 2002) 227 wären die Hellanodiken teilweise oder ausschließlich für Schiedsverfahren zwischen nichtLakedaimoniern zuständig, besonders auf den Feldzügen, da es schwierig gewesen wäre, jede Angelegenheit an die spatanischen Gerichtshöfe weiterzuleiten. Lipka nimmt ferner an, dass diese spartanischen Hellanodiken "directly connected" mit den homonymen Funktionären 
Symmachie betrifft, nimmt L. Thomsen an, dass dies nur nach den Perserkriegen sinnvoll gewesen wäre, während H. Schaefer und M. Lipka sie bereits in das 6. Jh. v. Chr. datieren. Da Lipka die von Xenophon erwähnten Hellanodiken sogar mit den gleichnamigen Funktionären von Olympia in Bezug bringt, soll hier jedoch nochmals betont werden, dass der Titel Hellanodike sowohl in literarischen Texten (3. Olympische Ode Pindars, 476 v. Chr.) als auch in Inschriften (erste Bezeugung im Zeitraum 475-450 v. Chr.) erst ab der Schlacht von Plataiai zu finden ist. ${ }^{10}$

Die Großmacht Sparta könnte freilich die originalen Titelträger von Olympia als panhellenische Richter innerhalb ihrer Symmachie gebraucht haben, da sie - besonders zur Zeit, da die Eleer ihre Bundesgenossen waren- das Heiligtum von Olympia als ein Werkzeug ihrer Politik innerhalb ihres Einflussbereiches ansah. Übrigens wäre es nicht das erste Mal, dass respektvolle Funktionären Olympias von den Spartanern in Bezug auf Kriegshandlungen in Anspruch genommen wurden: Herodot erzählt vom elischen Seher Tisamenos aus dem Iamidengeschlecht, den die Spartaner so hoch einschätzten und ihn als Oberpriester für den Kampf gegen Xerxes gewinnen wollten, dass er und sein Bruder Agias die einzigen Ausländer waren, die das spartanische Bürgerrecht erhielten. ${ }^{11}$ Ebenso möglich wäre es jedoch für Sparta, sich den Titel eines olympischen Funktionären anzueignen entweder in einer Periode der Symmachie mit den Eleern oder in einer Zeit elischspartanischer Feindlichkeit. $^{12}$

Mangels weiterer Angaben kann daher nicht mit Sicherheit beantwortet werden, ob die von Xenophon erwähnten Hellanodiken identisch mit den gleichnamigen Funktionären von Olympia sind oder es sich um den ersten Fall einer Imitation des Hellanodikentitels handelt. Für unsere Untersuchung ist jedoch wichtig, dass Sparta wegen der ideologischen Semiotik des Titels entweder bloß die Bezeichnung oder die originalen Hellanodiken als Richter in ihrer Symmachie gebrauchte, um dadurch zu betonen, daß sie alle Hellenen vertrat. Eine ähnliche Zielsetzung hatte übrigens auch der Titel Hellenotamiai der athenischen Symmachie.

von Olympia waren und der Titel des unparteiischen Schiedsrichters von den Spartanern in einer Zeit adoptiert wurde, als ihre Hegemonie noch nicht gefestigt war, d.h. in der zweiten Hälfte des 6. Jh. v. Chr.

10. Pind., Ol. 3. 12 und $I v O 2$, s. oben Anm. 3.

11. Hdt. 9. 33-36; vgl. auch S. Zoumbaki, Elis und Olympia in der Kaiserzeit. Das Leben einer Gesellschaft zwischen Stadt und Heiligtum auf prosopographischer Grundlage ( $« \mathrm{M} \varepsilon \lambda \varepsilon \tau \dot{\eta} \mu \alpha-$ $\tau \alpha \gg 32$; Athen 2001) 329-331, T 3.

12. Vgl. G. Schepens, «La guerra di Sparta contro Elide», in: E. Lanzilotta (Hrsg.), Ricerche di antiquità e tradizione classica (Tored 2004) 1-89. 
Die nächsten Bezeugungen des Titels außerhalb von Olympia hatten wahrscheinlich eine ähnliche ideologische Basis.

\section{Hellanodiken in Agonen außerhalb von Olympia}

Seit dem Anfang des 3. Jh.v. Chr. tritt der Hellanodikentitel in anderen Gebieten und in Bezug auf andere Agone auf. Die Bezeugungen werden im Folgenden kurz diskutiert. Schließlich wird der Versuch unternommen, die Fälle, wo der Hellanodikentitel entlehnt wurde, zu interpretieren und in einen historischen Rahmen zu stellen.

\section{Epidauros}

Kampfrichter unter dem Titel des Hellanodiken waren im Bereich des Rechtswesens in Epidauros aktiv und zwar in Fällen, die indirekt mit den Agonen zu tun hatten, wie eine Inschrift des 3. Jh. v. Chr. aus dem Stadion des Asklepieions bezeugt. In diesem Text erscheinen die Hellanodiken als eine dem Agonotheten untergeordnete Behörde. Der Agonothet und die Hellanodiken entscheiden, dass der

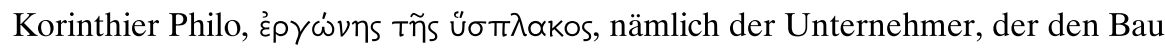
oder die Reparatur des Startpunktes des Stadions übernommen hatte, dem Heiligtum eine Geldsumme schuldig sei, da er offensichtlich seine Pflicht nicht regelmäßig durchgeführt hatte. Diese Entscheidung wurde vom Rat genehmigt. ${ }^{13}$ Die nächste Bezeugung von Hellanodiken in Epidauros ist viel später zu datieren: In einem Ehrendekret der Stadt für Euanthes, Sohn des Eunomos, ist unter anderen Ehrungen entschieden, daß der Geehrte mit einem Kranz geehrt wird, mit dessen Proklamation die Damiurgen, der Agonothet und die Hellanodiken beauftragt wurden,

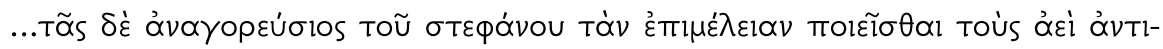

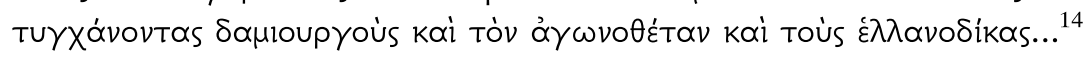

\section{Argos}

Die Proklamation der Ehrungen für Alexander aus Sikyon im Rahmen der Heraia und Nemea erscheint in einem Ehrendekret von Argos ebenfalls als eine Pflicht der Hellanodiken beider Agone. ${ }^{15}$ Zur Zeit des Dekrets wurden daher die Nemeischen

13. IG IV ${ }^{2}$ 1, 98. $\mathrm{Zu}$ einer Analyse der Einzelheiten der Prozesse s. K. HarterUibopuu, «The Asclepieia and Apolloneia of Epidauros. Malefactors at the sacred games»,

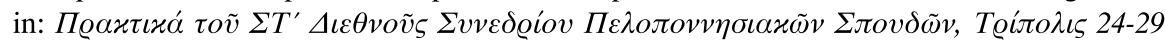
$\Sigma \varepsilon \pi \tau \varepsilon \mu$ ßoiov 2000, Bd II (Athen 2002) 330-340, bes. 330-334.

14. $I G \mathrm{IV}^{2} 1,66, \mathrm{Z} .55-59$.

15. W. Vollgraff, Mnemosyne 44 (1916) 64-71, Nr. III; s. auch Perlman, A. 24, 230-231 mit Literatur. 
Spiele nicht mehr von Kleonai, sondern von Argos organisiert. Gegen Anfang des 3. Jh. fand sogar die Veranstaltung der Nemeischen Spiele nicht mehr in Nemea, ${ }^{16}$ sondern in Argos statt; um diese Zeit wurde auch das Fest der Ekatombaia von Prosymna nach Argos übertragen und in Heraia umbenannt. Da die Nemea und Heraia kurz nacheinander stattfanden, bestimmte Argos für beide Feste gemeinsame zuständige Funktionäre, da mehrere weitere Inschriften bezeugen, dass nicht nur die Hellanodiken, sondern auch die Agonotheten und Theoroi ${ }^{17}$ sowie Thearodokoi ${ }^{18}$ beiden Veranstaltungen gemein waren.

Allgemein ist also der Text in das 3. Jh. zu datieren. Seine genaue Datierung ist jedoch Gegenstand langer Diskussion. Es handelt sich um einen Zeitraum, in dem Argos nicht vom Achäischen Bund kontrolliert wurde, denn es gibt keine Erwähnung eines Bundesmagistraten. So ist die Inschrift entweder vor $229 \mathrm{v}$. Chr. oder zwischen 225 und 215 v. Chr. zu setzen. Die Zeiträume 251-249 und 245-229 v. Chr. sind allerdings eher auszuschließen, da Argos und die Heimat des Geehrten, Sikyon, feindlichen Bünden, nämlich der achäischen und makedonischen Symmachie, angehörten. Der Text kann daher entweder in die erste Hälfte des 3. Jh. oder in den kurzen Zeitraum 225-215 v. Chr. datiert werden. ${ }^{19}$

Die nächsten Bezeugungen des Hellanodikentitels in Argos stammen aus der römischen Zeit. Eine Liste von Funktionären, die sich auf Agone beziehen (2./3. Jh. n. Chr.), und eine Ehreninschrift für den Agonotheten der Sebasteia und Nemea, die von den Hellanodiken aufgestellt wurde (2. Jh. n. Chr.), weisen darauf hin, dass das Hellanodikenkollegium in der römischen Zeit aus zehn Mitgliedern zusammengesetzt war und dass es noch einen - wahrscheinlich den Hellanodiken übergeordneten- Agonotheten gab. ${ }^{20}$

16. Zur wechselnden Kontrolle über die Nemeischen Spiele s. Perlman, 138-149 und 155 mit älterer Literatur. M. Mari, Al di là dell'Olimpo. Macedoni e grandi santuari della Grecia dall'età arcaica al primo ellenismo ( $\ll \varepsilon \overline{\mathrm{M}} \varepsilon \tau \dot{\eta} \mu \alpha \tau \alpha » 34$; Athen 2002) $189 \mathrm{ff}$.

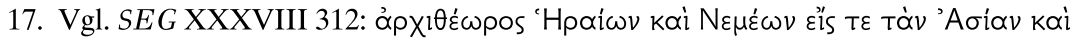
हi่ 'A $A \lambda \varepsilon \xi \alpha \dot{\alpha} \nu \delta \rho \varepsilon l \alpha \nu$.

18. Perlman, 132 Anm. 143.

19. Zu einer Zusammenfassung der verschiedenen Datierungsvorschläge s. P. Amandry, «Sur les concours argiennes», in: Études argiennes, BCH Suppl. VI (1980) 226-227, Anm.

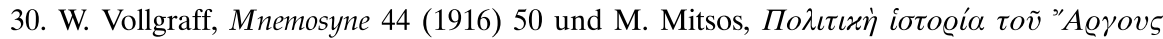
(Athen 1945) 70 datieren den Text in die erste Hälfte des 3. Jh., Charneux allgemein in das 3. Jh., Amandry in die erste Hälfte des 3. Jh. oder zwischen 225 und 215 v. Chr.

20. Für die Liste s. IG IV 587 und P. Charneux, $B C H 80$ (1956) 608 und für die Ehreninschrift s. ders., $B C H 80$ (1956) 604-10, no. 6 (SEG XVI 253). 


\section{Korinth}

Erst in der römischen Zeit sind Hellanodiken in Bezug auf das dritte in der Peloponnes veranstaltete panhellenische Fest, nämlich Isthmia, bezeugt. Die älteste dieser Bezeugungen ist eine Siegerliste erst aus dem Jahr 3 n. Chr., wo der Agonothet und die Hellanodiken zuoberst im Katalogs aufgezählt sind. ${ }^{21}$ Eine Serie derartiger Listen macht ferner klar, dass das Kollegium hier ebenso zehn Mitglieder inkludierte. Die Hellanodiken als Körperschaft ehren ferner den Agonotheten der Isthmischen Spiele, der in der Hierarchie höher zu stehen scheint. ${ }^{22}$ Besonders interessant ist übrigens eine Gruppe von Bronzetafeln aus dem Poseidonheiligtum am Isthmos, auf denen Namen von Athleten stehen, die von den Hellanodiken von einer Teilnahme an den Spielen ausgeschlossen wurden. ${ }^{23}$

Wann die Hellanodiken in Isthmia eingeführt wurden, ist unbekannt; kein Beleg ist in die vorrömische Zeit zu datieren. Allen Brown West hält die Hellanodiken, genauso wie die Hieromnemonen und die Agonotheten, für Überbleibsel aus der griechischen Vergangenheit von Korinth, da es sich um gänzlich fremde Institutionen für eine römische Kolonie handelt. ${ }^{24}$ Nichtsdestoweniger kommen die Hellanodiken von Isthmia schon in den ersten Jahren der Kaiserzeit vor, und so könnten sie kaum als eine der zahlreichen Kopien der Bezeichnung in den Olympischen und Isolympischen Spielen der römischen Zeit angesehen werden, die später häufig bezeugt sind. ${ }^{25}$ Es scheint daher trotz mangelnden Quellenmaterials nicht unmöglich, dass die Hellanodiken in der hellenistischen Zeit in Isthmia eingeführt wurden, vielleicht schon im 3. Jh. v. Chr., d.h. gleichzeitig mit den ersten Bezeugungen des Kollegiums in Argos und Epidauros.

21. Corinth VIII $1,14$.

22. Vgl. z. B. O. Broneer, «Isthmia excavations, 1952», Hesperia 22 (1953) 192.

23. D.R. Jordan $\mathcal{E}$ A.J.S. Spawforth, «A new document from the Isthmian games», Hesperia 51 (1982) 65-68 (SEG XXXII 364; Bullépigr 1982, 173) und D.R. Jordan, «Inscribed lead tablets from the games in the sanctuary of Poseidon», Hesperia 63 (1994) 111-116. Die Überprüfung der Athleten fand wahrscheinlich in den sog. દ̇ $\gamma$ kpıтท́piol oíkol statt, vgl. IG IV 203; vgl. Jordan, a.O., 112, 115.

24. $\mathrm{Zu}$ den Argumenten von West s. Kommentar in Corinth VIII 2, 81. Der gleichen Meinung ist auch A. Hupfloher, «A small copy of Rome? Religious organisation in Roman Corinth», in: A.D. Rizakis \& Fr. Camia (Hrsgg.), Pathways to power. Civic elites in the eastern part of the Roman Empire (Athens 2008) 159.

25. A. Farrington, «Olympic victors and the popularity of the Olympic games in the Imperial period», Tyche 12 (1997) 15-46. 
Der historische Rahmen der Bezeugungen von Hellanodiken im 3. Jh. v. Chr. Im Laufen des 3. Jh. v. Chr. findet sich also der Hellanodikentitel in Zusammenhang mit wichtigen Festen der Peloponnes auf, d.h. sowohl in Epidauros als auch in Argos und vielleicht in Korinth. In den beiden erstgenannten Städten sind in dieser Periode intensive Bautätigkeit und Erhöhung des Status ihrer athletischen Veranstaltungen zu beobachten. Es ist übrigens charakteristisch, dass sich beide Städte bemühen, als Organisatoren von panhellenischen Agonen zu erscheinen, Argos der Nemeischen Spiele und Epidauros ihrer lokalen Agone, die nun wie panhellenische Spiele präsentiert wurden. Die Vollendung des Theaterbaus in Argos um die Mitte der 270er Jahre wird sogar von manchen Forschern in Zusammenhang mit der Übernahme der Heraia und Nemea durch die Stadt gebracht; ${ }^{26}$ die erste Bezeugung des Hellanodikentitels zeigt, wie gesagt, dass die Funktionäre beiden Veranstaltungen gemein waren. Was Epidauros betrifft, erlebt das Asklepiosheiligtum schon vom Ende des 4. Jh. v. Chr. und während des 3. Jh. v. Chr. eine ungeheure Blüte, wie die große Bautätigkeit und die Erhöhung der ursprünglich lokalen Asklepieia und Apolloneia zu panhellenischen Spielen beweisen. ${ }^{27}$ Es liegt daher die Vermutung nahe, dass der symbolträchtige Hellanodikentitel mehr oder weniger gleichzeitig in die Agone beider Städte, Argos und Epidauros, eingeführt wurde, um den Richtern zusätzliche Rechtsgültigkeit, den Veranstaltungen zusätzliche Ausstrahlungskraft und den organisierenden Städten zusätzliches Prestige zu bieten.

\section{Heiligtümer und Festlichkeiten als Instrument der politischen Propaganda}

Dass die prominenten Heiligtümer und Feste wichtige Instrumente für die Förde-

26. Vgl. J.-Ch. Moretti (avec la collaboration de St. Diez), Thêatres d'Argos (Paris 1993) 8-23, wo die Vollendung des Theaters in das erste Viertel des 3. Jh.v. Chr. datiert wird; E. Barakari \& A. Pariente, «Argos du VIIe au IIe siècle av. J.C.: Synthèse des données archéologiques», in: A. Pariente $\mathcal{E}$ G. Touchais (Hrsgg.), Argos et l'Argolide, Topographie et urbanisme, Actes de la table ronde internationale Athènes-Argos 28/4-1/5/1990 (Athènes 1998) 170 datieren das Theater in das erste Viertel des 3. Jh. und bringen dies in Zusammenhang mit der Übertragung der Spiele. Vgl. auch Amandry, a.O. (Anm. 19), 245-7; J.-Ch. Moretti, «L'implantation du theâtre d'Argos dans un lieu plein de sanctuaires», in: A. Pariente et G. Touchais (Hrsg.), Argos et l'Argolide, a.O., 233-259; Perlman, n. 149.

27. Zur Bautätigkeit vgl. die diesbezüglichen Abrechnungen, s. L. Gounaropoulou, Die Bauabrechnung des Asklepiostempels in Epidauros. Ein archäologischer Kommentar (Diss. Univ. Wien 1983); zur panhellenischen Ausstrahlung der Spiele vgl. die Thearodokenlisten, s. IG IV $24-95$ und Perlman, $67 \mathrm{ff}$. 
rung einer Ideologie oder politischen Propaganda bildeten, ist wohl bekannt. ${ }^{28}$ Die Frage, die sich dabei erhebt, betrifft den politischen Symbolgehalt der Einführung des Hellanodikenkollegiums in die obenerwähnten Veranstaltungen. Da diese Einführung sogar in die gleiche Periode zu datieren scheint, dürfte man an eine Übermacht denken, die damals sowohl Argos als auch Epidauros sowie vielleicht Korinth kontrollierte und einen Grund hätte, das Hellanodikenkollegium in diese Agone einzuführen.

Die Übermacht, die während dieser Periode alle obenerwähnten Städte der Nordostpeloponnes auf ihre Seite brachte, waren die Makedonen. Epidauros, zusammen mit Troezen und Megara, gehörte zum Einflussbereich der Makedonen bis die Stadt 243 v. Chr. dem Achäischen Bund beitrat; Demetrios Poliorketes hat sogar Truppen aus der Nordostpeloponnes eingesetzt, was inschriftlich mindestens für Troezen ( $I G$ IV 750) überliefert wird, da Gefangene aus der Stadt von Lysimachos (287 v. Chr.) erwähnt werden. J. Gabbert nimmt sogar an, dass es eine makedonische Garnison in Epidauros gab. ${ }^{29}$ Was Argos betrifft, das erst 229 v. Chr. dem Achäischen Bund beitrat, gab es in der Stadt einen starken promakedonischen Kern. 303 v. Chr. stellte Demetrios Poliorketes Argos unter seine Kontrolle (Plut. Dem. 25. 1-2). In Argos setzte Antigonos Gonatas einen Tyrannen ein, so auch in Elis und Megalopolis. ${ }^{30}$ Darüber hinaus bildete das Gebiet des Isthmos einen der wichtigsten, vielleicht den wichtigsten, makedonischen Stützpunkt in Südgriechenland, ${ }^{31}$ nachdem Demetrios Poliorketes 303 v. Chr. in Korinth - das von den Ptolemäern (308 v. Chr.) besetzt worden war- eine Garnison errichten konnte. Korinth bildete zusammen mit Demetrias und Chalkis die Bollwerke der makedonischen Macht in Griechenland. ${ }^{32}$ Generell war das ganze Nordostpeloponnes unter makedonischer Kontrolle.

Das Verhältnis der Makedonen zu den Heiligtümern von Südgriechenland und der Einsatz ihrer Agonen und Feste zum Zwecke makedonischer Propaganda haben ihren Ursprung in älteren Zeiten. ${ }^{33}$ In der Periode, die für unsere Forschung

28. Insbesondere zum Thema in hellenistischer Zeit s. D. Di Nani, Concorsi sportivi e propaganda in età ellenistica (Diss. Napoli 2006).

29. Gabbert, Antigonus II Gonatas, 33.

30. W.W. Tarn, Antigonos Gonatas (Nachdr. Chicago 1969) 272 ff. Vgl. Polybios 2. 41, 10; 9. 29, 6 zu den Tyrannien, die Antigonos Gonatas in verschiedenen Städten "pflanzte".

31. Vgl. J.B. Scholten, «Macedon and the mainland», in: A. Erskine (Hrsg.), A companion to the Hellenistic world (Oxford 2007 $\left.7^{3}\right) 143 \mathrm{ff}$.

32. s. Gabbert, Antigonus II Gonatas, 33-34.

33. M. Mari, Al di là dell'Olimpo. Macedoni e grandi santuari della Grecia dall'età arcaica 
in Betracht kommt, haben die Antigoniden in verschiedenen Fällen die Heiligtümer und die Feste der Peloponnes als Werkzeug ihrer Politik verwendet. Im Jahre 303 übernahm Demetrios Poliorketes die Proedrie der Heraia (Plut. Dem. 25. 1-2), nachdem er Argos unter seine Kontrolle gestellt hatte, und gleichzeitig feierte er seine Heirat mit Deidameia, der Schwester von Pyrrhos und einer Verwandten Olympias, und unterstrich somit die politische und symbolische Bedeutung dieser Heirat. Bald darauf, bei den Isthmien des Jahres 302, hat er Gesandte aller von ihm

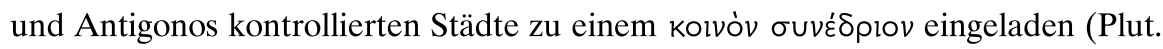
Dem. 25. 3), was den ersten Schritt in Richtung des von ihm geplanten Hellenenbundes, praktisch eine Neugründung des Korinthischen Bundes, bildete. ${ }^{34}$ Die Wahl von Korinth und Isthmia als Schauplatz dieses Synedrions, dessen Satzung in einer fragmentarischen Inschrift aus Epidauros ${ }^{35}$ erhalten ist, strebt nach Anerkennung der Antigoniden als rechtmäßige Nachfolger von Philipp und Alexander.

Die übrigen Mächte jener Periode übten ähnliche Praktiken, nämlich den Gebrauch von wichtigen religiösen Zentren und panhellenischen Festen oder Erhebung lokaler Veranstaltungen zu panhellenischem Status als Instrumente der Förderung ihrer Propaganda. Es gibt zahlreiche Beispiele dieser Verfahrensweise. So strebt Ptolemaios Philadelphos nach Anerkennung der Agone, die er zum Andenken an seinen Vater, Ptolemaios Soter, veranstaltete und zu denen er die griechischen Städte eingeladen hatte, als isolympisch. ${ }^{36} \mathrm{~L}$. Robert hat betont, dass diese

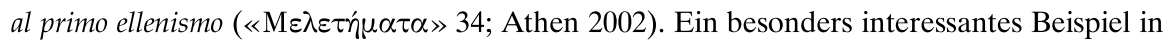
dieser Hinsicht ist die Stiftung des Philippeions in Olympia durch Philipp II., s. Mari, $183 \mathrm{ff}$.

34. Zum von den Antigoniden neugegründeten Hellenenbund und zur Einordnung des Nesiotenbundes in diesen erweiterten Bund s. Buraselis, Das hellenistische Makedonien, 85-87.

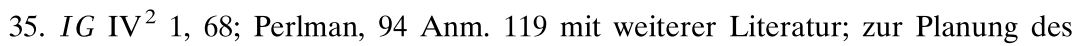
Synedrion gleichzeitig mit den Isthmien s. Perlman, 94-95.

36. Vgl. Syll ${ }^{3} 390=I G$ XII 7, 506 Dekret des Nesiotenbundes von Nikouria, eine kleine Insel in der Nähe von Amorgos; FD III 4, 357 Dekret von Delphi über Ptolemaia.

Es gibt verschiedene Datierungsvorschläge für die Gründung des Ptolemäen-Festes, die zwischen dem Ende der 280er Jahre (z.B. R.A. Nerwinski, The foundation date of the Panhellenic Ptolemaia and related problems in early Ptolemaic chronology [Diss. Univ. Duke 1981] 30 ff., setzt die erste Feier 282 v. Chr. an) und dem Ende der 260er Jahre (R.A. Hazzard, Imagimation of a monarchy: Studies in Ptolemaic propaganda [Toronto 2000] 57-58 mit der älteren Literatur) schwanken. Chr. Habicht, «Athens and the Ptolemies», in: Athen in hellenistischer Zeit. Gesammelte Aufsätze (München 1994) 142 und Anm. 10 nimmt an, daß die ersten Ptolemaia 282 v. Chr. als "one-time event" gedacht waren; 280/79 v. Chr. Dann entschied Ptolemaios, die Ptolemaia als ein penteterisches Fest zu veranstalten. Zur Diskus- 
Aktion des Ptolemaios eine neue Tendenz kennzeichnete, nämlich der Vermehrung der Agone, die in der darauffolgenden Zeit zu panhellenischem Status erhoben werden. ${ }^{37}$ Darüber hinaus sind hier die Donationen seitens des Ptolemaios II für die Größeren Panathenaea nach der Befreiung Athens zu erwähnen, die im Kalliasdekret (Z. 64-70) bezeugt sind. ${ }^{38}$ Heiligtümer und Feste werden ferner als

sion der Chronologie aufgrund des athenischen Kalliasdekretes und in Bezug auf die erste Ausführung der Großen Panathenaia nach der Befreiung Athens von der makedonischen Herrschaft s. unten Anm. 38.

Damit wird von manchen Forschern die große Prozession des Festes in Bezug gebracht, die vom Rhodier Kallixeinos bei Athenaios, 5. $197 \mathrm{D}$ ff. beschrieben wird. In dieser Prozession, die die Macht und den Reichtum des Königs darstellte, befand sich neben Götterbildern und Statuen von Alexander und Ptolemaios Soter eine Allegorie der Stadt Korinth. Eine Forschungstendenz sieht einen Sinn in dieser Allegorie nur in einer Periode, in der Korinth sich unter ptolemäischem Einfluß befand, nämlich ab 308 v.Chr., als Ptolemaios I. den Hellenenbund neu zu gründen versuchte. Eine andere Ansicht wurde von E. Rice, The grand procession of Ptolemy Philadelphus (Oxford 1983) 102-109 unterstützt und von K. Buraselis, «Ambivalent roles of centre and periphery. Remarks on the relation of the cities of Greece with the Ptolemies until the end of Philometor's age», in: P. Bilde et al. (Hrsgg.), Centre and periphery in the Hellenistic world (Aarhus 1996) 262 angenommen, dass diese Allegorie nur als eine Erinnerung dieser Bemühungen von Ptolemaios I. im Rahmen der ptolemäischen Propaganda sinnvoll zu interpretieren sei, die noch viele Jahrzehnte überlebte; zu den Ereignissen des Jahres 308 v. Chr. Buraselis, Das hellenistische Makedonien, 49. Hierbei ist vielleicht die Ansicht von V. Foertmeyer, «The dating of the pompe of Ptolemy II Philadelphus», Historia 37 (1988) 90-104 zu erwähnen, die die große Parade der Ptolemaia als "mobilisation for the war, rather than...a celebration for its success" ansieht.

37. L. Robert, OMS VI, 709-719. Die Ansicht von Robert wird von R. Parker, «New 'Panhellenic' festivals in Hellenistic Greece», in: R. Schlesier $\mathcal{E}$ U. Zellmann (Hrsgg.), Mobility and travel in the Mediterranean from Antiquity to the Middle Ages (Münster 2004) 9-22 in Abrede gestellt, da es sich nur um eine Mode von isolympischen, isopythischen usw. Agonen handelt, die mehrheitlich in viel älteren Zeiten gegründet wurden.

38. Zum Ehrendekret für Kallias von Sphettos s. T.L. Shear, J.R., Kallias of Sphettus and the revolt of Athens in 286 B.C., Hesperia Supplement 17 (Princeton 1978) (SEG XXVIII $60 ; 29,102)$, sowie P. Paschidis, Between city and the king. Prosopographical studies on the intermediaries between cities of the Greek mainland and the Aegean and the royal courts in the

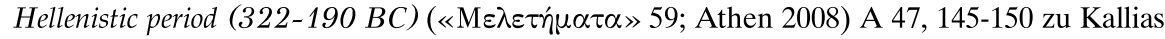
und zu einer Zusammenfassung der Literatur; zur Chronologie der ersten Ausführung der Großen Panathenaia nach der Befreiung Athens von der makedonischen Herrschaft s. Chr. Habicht, «Athens and the Ptolemies», in: Athen in hellenistischer Zeit. Gesammelte Aufsätze (München 1994) 142 und Anm. 10, der die Ansicht von Nerwinski, a.O. (Anm. 36) 30-41 teilt; vgl. ferner die Gegenargumente von B. Dreyer, Untersuchungen zur Geschichte des spätklassischen Athen (322-ca. 230 v. Chr.) (Stuttgart 1999) $206 \mathrm{ff}$. 
politisches Instrument von den Aitolern gebraucht, die Delphi kontrollierten und darüber hinaus um den Anfang der 240er Jahre die Soteria mit einer großen Pracht begingen, da dieses Fest mit dem wichtigsten Ereignis ihrer Geschichte, nämlich der Zurückschlagung der Galater, verbunden war. ${ }^{39}$

\section{Die Begriffe "Hellas" und "Hellenes" in der politischen Propaganda}

Jede Macht strebt also danach, wichtige panhellenische Heiligtümer und Veranstaltungen zugunsten ihrer Politik einzusetzen und jede Gelegenheit zu ergreifen, sich als rechtsmäßiger Vertreter aller Hellenen zu präsentieren. Die Begriffe Hellas und Hellenes und ihre Derivaten haben besonders ab der Gründung des panhellenischen Bundes durch Philipp II. einen intensiven Symbolcharakter und eine politische Semiotik angenommen und wurden deswegen von präsumtiven Aussprechern aller Hellenen, Ptolemäer, Aitoler, Antigoniden, im Streit über ihre Einflusssphären gebraucht. Es ist charakteristisch beispielsweise, dass die delphische Amphiktyonie ab dem ersten Viertel des 3. Jh. v. Chr., d.h. in der Periode der aitolischen Kontrolle, oft nicht bloß im Namen der Amphiktyonie und der übrigen Hellenen, sondern im Namen aller Hellenen spricht. ${ }^{40}$ Eine ähnliche Bestrebung der Ptolemäer, sich in der Tradition des Panhellenismus zu präsentieren, ist an mehreren Aktionen zu erkennen, wie z.B. in der Planung, den Hellenenbund bei den von Ptolemaios 308 v. Chr. als Feier der griechischen Befreiung veranstalteten Isthmia neu zu gründen, ${ }^{41}$ oder später in der Rhetorik von Chremonides, der von

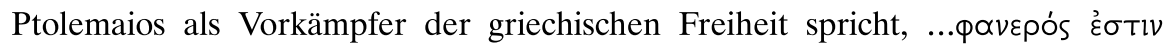

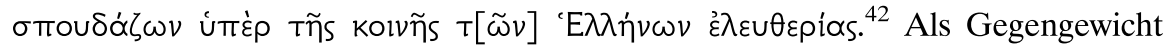
zielte die Expedition von Antigonos 307 v. Chr. auf "Befreiung der Griechen"

39. Die Soteria werden nun vom aitolischen Agonotheten veranstaltet; ihr musischer Agon wird zum Status der Pythia und der gymnische und hippische zum Status der Nemea erhöht; es gibt auch den Vorschlag, dass nun Soteria als ein penteterisches Fest gefeiert wurden, s. J. Scholten, The politics of plunder (Berkeley 2000) 99-100 und 238 und Anm. 9-10.

40. Die wichtigsten Beispiele dieser Terminologie der Amphiktyonie diskutieren F. Lefèvre, L'amphictionie pyléo-delphique: Histoire et institutions (Paris 1998) 163, 180 und J.-L. Ferrary, «Rome et la géographie de l'hellénisme: réflexions sur "hellènes" et "panhellènes" dans les inscriptions d'époque romaine», in: O. Salomies (Hrsg.), The Greek East in the Roman Contex. Proceedings of a Colloquium Organised by the Finnish Institute at Athens, May 21 and 22, 1999 (Helsinki 2001) 20-21.

41. Vgl. Suda, s.v. Demetrios; ferner s. Buraselis, Das hellenistische Makedonien, 51 und Anm. 51.

42. Syll ${ }^{3}$ 434-435, Z. 17-18. Vgl. R.A. Hazzard, Imagination of a monarchy: Studies in Ptolemaic propaganda (Toronto 2000) 57 und Anm. 48. 
$\mathrm{ab} ;{ }^{43}$ hierbei ist nochmals der obenerwähnte makedonische Plan zu erwähnen, den panhellenischen Bund (302 v. Chr.) neu zu gründen und im Namen aller Hellenen zu entscheiden.

\section{Die Bedeutung des Hellanodikentitels im Rahmen der politischen Propaganda der Großmächte des 3. Jh. v. Chr.}

Der Titel des Hellanodiken war in diesem Rahmen, wie bereits zuvor innerhalb der Peloponnesischen Symmachie, als respektvoller Träger mit Symbolcharakter von langer Tradition bedeutend. Wenn er tatsächlich von den Makedonen auf peloponnesischen Feste außerhalb Olympias eingeführt wurde, als die dafür verantwortlichen Städte auf makedonischer Seite standen, lohnte es sich festzuhalten, dass gerade in dieser Periode Olympia, bzw. Elis, vom makedonischen zum aitolischen Einflusskreis wechselte. Dies passierte mit der Ermordung des von Antigonos Gonatas durchgesetzten Tyrannen von Elis, Aristotimos (272/271 v. Chr.), was vielleicht durch aitolische Unterstützung ermöglicht wurde. ${ }^{44}$ Kyllon, der den Tyrannen ermordet hatte, wurde von den Aitolern in Olympia und Delphi geehrt, was die Bedeutung der Gewinnung von Elis und Olympia für die Aitoler unterstreicht. ${ }^{45}$ Ebenso schwerwiegend dürfte der Verlust für die Makedonen gewesen sein. So wollte möglicherweise Antigonos Gonatas mittels der Benennung der Richter der von ihm kontrollierten Agonen zu Hellanodiken betonen, dass er weiterhin über das Schicksal der Hellenen entschied, besonders nach dem Tod des Pyrrhos in Argos (272 v. Chr.) und nach der Durchsetzung von Antigonos im Chremonideischen Krieg in den frühen 260er Jahren, als sich die makedonische Macht durch siegreiche Kampfhandlungen auf dem Isthmos und in Attika erneut stabilisieren konnte. ${ }^{46}$

Dass der Hellanodikentitel ohne Vorbehaltung in andere Agonen eingeführt worden sein könnte, zeigen andere ähnliche Ereignisse. Es lohnt sich etwa, an die Veranstaltung von Pythia in Athen 290 v. Chr zu erinnern, da Demetrios Polior-

43. Buraselis, Das hellenistische Makedonien, $52 \mathrm{ff}$.

44. Zu Tyrannen, die Antigonos Gonatas in Argos, Megalopolis, Elis und vielleicht auch in Epidauros durchgesetzt hat s. Gabbert, Antigonus II Gonatas, 33. Zu Aristotimos s. S.

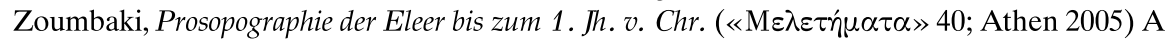
114; zu diesen Ereignissen s. F. J. Gómez Espelosín, «Plutarch and Justin on Aristotimus of Elis», AJPh 112 (1991) 103-109; C. Bearzot, Storia e storiografia ellenistica in Pausania il Periegeta (Venezia 1992) $143 \mathrm{ff}$.

45. s. W.W. Tarn, Antigonos Gonatas (Nachdruck Chicago 1969) 287-288.

46. Gabbert, Antigonus II Gonatas, 45 ff. 
ketes nämlich veranlasst hatte, dass die Spiele auf einen anderen Ort zu verlegen seien, zumal damals Delphi und das panhellenische Fest auf der Seiten der Aitoler standen. ${ }^{47}$ Es läßt sich freilich nicht beweisen, daß die Einführung des Hellanodikentitels in Veranstaltungen unter makedonischer Kontrolle gerade nach dem Verlust von Olympia für die Makedonen zu datieren sei, da die diesbezüglichen Belege nicht so präzis datierbar sind. Es könnte allerdings jederzeit stattgefunden haben und zwar unverhohlen, wie die allgemeine Politik der Makedonen in derartigen Angelegenheiten zeigt.

Dass die Hellanodiken in den athletischen Veranstaltungen außerhalb Olympias hauptsächlich eine symbolische Rolle spielten, bezeugt die Tatsache, dass sie in die schon existierende Struktur der agonistischen Organisation eingeführt wurden, ohne eine wesentliche Änderung hervorzurufen. In diesen Agonen waren die Hellanodiken dem Agonotheten untergeordnet. Darüber hinaus, scheint hier die Bekleidung der Hellanodikenfunktion mit keiner besonderen sozialen Stellung verbunden gewesen zu sein, da kein Hellanodike dieser Agone der hellenistischen Zeit namentlich überliefert ist und in keiner weiteren Inschrift vorkommt. Anders liegt der Fall in Olympia, wo die Hellanodiken prominente Personen waren und sie sogar für eine Zeit lang als eponyme Beamten fungierten. ${ }^{48}$

In der Kaiserzeit überlebt das Prestige der Hellanodikenfunktion nicht einmal in Olympia. Ehreninschriften für Hellanodiken sind nicht mehr zu finden; ${ }^{49}$ das Hellanodikenamt kommt nicht im cursus honorum vor, was verrät, dass es nicht jenen Ämtern angehörte, die prominenten Leuten vorbehalten wurden. Der Titel hatte anscheinend im Rahmen der römischen Herrschaft das ideologische Gewicht der früheren Epochen eingebüßt.

47. Plut. Dem. 40. 7-8; Chr. Habicht, Athen. Die Geschichte der Stadt in hellenistischer Zeit (München 1995) 100.

48. Zur Nennung von Hellanodiken als Datierungsangabe in einem pisatischen Dekret s. IvO 36 (365-363 v. Chr.). Zur Bedeutung des Hellanodikenamtes in Eleia s. S. Zoumbaki, Elis und Olympia in der Kaiserzeit. Das Leben einer Gesellschaft zwischen Stadt

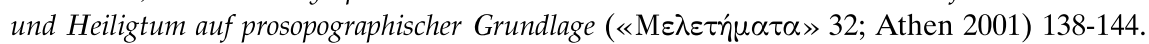

49. Die letzte erhaltene Ehreninschrift für einen Hellanodiken in Olympia, IvO 422, stammt aus der augusteischen Zeit. 